\title{
Model of charge and magnetic order formation in itinerant electron systems
}

\author{
Romuald Lemański \\ Institute of Low Temperature and Structure Research, \\ Polish Academy of Sciences, Wroctaw, Poland
}

\begin{abstract}
We propose a simple model of charge and/or magnetic order formation in systems containing both localized and itinerant electrons coupled by the on-site, spin-dependent interaction that represents Coulomb repulsion and Hund's rule (a generalized Falicov-Kimball model). Ground state properties of the model are analyzed on the square lattice on a basis of the phase diagrams that have been constructed rigorously, but in a restricted configurational space. For intermediate values of the coupling constants there are considerable ranges of itinerant electron densities where phases with complex charge and magnetic structures of the localized electrons have lower energy than the simplest antiferro- and ferromagnetic ones. A strong tendency towards the antiferromagnetic coupling between spins of localized electrons has been observed close to half-filling for any density of localized electrons, including situations where the magnetic ions are diluted. For small band fillings the ferromagnetic coupling between localized spins is predominant.
\end{abstract}




\section{INTRODUCTION}

The purpose of this contribution is to introduce a simple model of charge and/or magnetic order formation generated by an on-site, spin-dependent interaction of itinerant electrons with localized ones, and to provide a preliminary analysis of that model. The model is able to capture many essential aspects of magnetism of both the localized and itinerant electrons. It is based on the Falicov-Kimball model (FKM), which was originally proposed as a model of metal-insulator transitions in mixed-valence compounds ${ }^{1}$. Later on the FKM appeared to be suitable for studying some other phenomena as well, as e.g. a tendency for charge-density-wave formation in interacting fermion systems ${ }^{2-4}$. Actually, in most of applications studied so far the spinless version of the model was explored. As far as we know, spin degrees of freedom were taken into account only in a few papers ${ }^{5-11}$, but in all of them the interactions between electrons were spin-independent.

However, many experiments show that a charge superstructure often occurs together with a magnetic order ${ }^{12-14}$. In order to describe both within a single model we proposed a generalization of the FKM with an anisotropic, spin-dependent local interaction. These ideas have also been applied to the Hubbard model ${ }^{15}$, but that work has been performed only in the limit of a large spin coupling constant; the authors named this model the "2-band Ising-Hubbard model". Hence we call our model the Ising-Falicov-Kimball model.

The model Hamiltonian is

$$
\begin{aligned}
& H=\sum_{<m, n>} \sum_{\sigma=\uparrow, \downarrow}(t-\left.\mu_{d} \delta_{n m}\right) d_{m, \sigma}^{+} d_{n, \sigma}+\left(E_{f}-\mu_{f}\right) \sum_{m} \sum_{\eta=\uparrow, \downarrow} f_{m, \eta}^{+} f_{m, \eta} \\
&+U \sum_{m} \sum_{\sigma, \eta=\uparrow, \downarrow} d_{m, \sigma}^{+} d_{m, \sigma} f_{m, \eta}^{+} f_{m, \eta} \\
&+J \sum_{m} \sum_{\sigma=\uparrow, \downarrow}\left(d_{m, \sigma}^{+} d_{m, \sigma} f_{m,-\sigma}^{+} f_{m,-\sigma}-d_{m, \sigma}^{+} d_{m, \sigma} f_{m, \sigma}^{+} f_{m, \sigma}\right),
\end{aligned}
$$

where $\langle m, n>$ means the nearest neighbor lattice sites $m$ and $n, \sigma$ and $\eta$ are spin $-1 / 2$ indices, $d_{m, \sigma}\left(f_{m, \sigma}\right)$ and $d_{m, \sigma}^{+}\left(f_{m, \sigma}^{+}\right)$are annihilation and creation operators of itinerant (localized) electrons, respectively. The on-site interaction between localized and itinerant electrons is represented by two coupling constants: $U$, which is spin-independent Coulombtype and $J$, which is spin-dependent Ising-type. The later reflects the Hund's rule force. The other parameters of the model are: the hopping amplitude $t$ (we will set it equal to one for our energy scale), the energy level of localized electrons $E_{f}$ and the chemical potentials of itinerant $\mu_{d}$ and localized $\mu_{f}$ electrons, respectively. Here $E_{f}$ and $\mu_{f}$ are given as independent parameters, but physical properties of the system depend only on the difference $\mu_{f}-E_{f}$. Taking into consideration that fact, the ground canonical phase diagram was chosen to be displayed in the variables $\mu_{d}$ and $\mu_{f}$, as if $E_{f}$ would be equal to 0 . However, it is equivalent to another representation with $\mu$ and $\mu-E_{f}$ as independent parameters (if one puts $\mu_{d}=\mu_{f}=\mu$ ).

Double occupancy of the localized electrons is forbidden, implying the on-site Coulomb repulsion $U_{f f}$ between two $f$-electrons is infinite. Consequently, at a given site the $f$-electron occupancy is assumed to be $n_{f}=n_{f, \uparrow}+n_{f, \downarrow} \leq 1$ and the $d$-electron occupancy to be $n_{d}=n_{d, \uparrow}+n_{d, \downarrow} \leq 2$. So there are 3 states per site allowed for the $f$-electrons $\left(n_{f}=0\right.$;

$n_{f, \uparrow}=1$ and $n_{f, \downarrow}=0 ; n_{f, \uparrow}=0$ and $\left.n_{f, \downarrow}=1\right)$ and 4 states per site allowed for the $d$-electrons $\left(n_{d}=0 ; n_{d, \uparrow}=1\right.$ and $n_{d, \downarrow}=0 ; n_{d, \uparrow}=0$ and $\left.n_{d, \downarrow}=1 ; n_{d}=2\right)$. 
The first three terms of the Hamiltonian (1) form the multicomponent FKM studied recently in the $2 D$ case $^{10}$ and in the limit of infinite dimensions ${ }^{8,9}$. The last term of (1) describes a simplified interaction that couples spins of an itinerant and localized electron occupying the same site. The inclusion of this interaction enables one to describe magnetic structures (in addition to charged ones) produced by localized electrons and, at the same time, the band magnetism of itinerant electrons.

All single-ion interactions included in (1) preserve states of the localized electrons, i.e. the itinerant electrons traveling through the lattice change neither occupation numbers nor spins of the localized ones. In other words, $\left[H, f_{i \eta}^{+} f_{i \eta}\right]=0$ for all $i$ and $\eta$, so the local occupation number is unchanged. This is a characteristic feature of the Falicov-Kimball based models, which makes them tractable in a controllable way.

The localized electrons play the role of an external, charge and spin dependent potential for the itinerant electrons. This external potential is "adjusted" by annealing, so the total energy of the system attains its minimum. So there is a feedback between the subsystems of localized and itinerant electrons, and this is the feedback that is responsible for the longrange ordered arrangements of the localized ones, and consequently for the formation of various charge and/or spin distributions in low temperatures.

On the other hand, if the total magnetization of the localized electrons is non-zero, the potential experienced by the itinerant spin-up electrons differs from that of spin-down ones. As a result, the spins of the itinerant electrons become partially polarized, so we also have unsaturated band magnetism.

So far it was a common practice to investigate the isotropic, Heisenberg-type interaction between spins of localized and itinerant electrons (e.g. within a framework of the $s-d$ model ${ }^{16}$ ). Here, instead, we propose an Ising-type coupling between the spins (see also Ref. ${ }^{15}$ ). An advantage of the latter approach is that the model can be treated rigorously. However, one can also provide a plausible physical background that justifies the assumption that the spin-flip processes generated by $S^{+} S^{-}$and $S^{-} S^{+}$operators, which are characteristic for the Heisenberg-type interaction, can be neglected in a first approximation. The reasoning comes from a simple notice that magnetic structures observed in many materials are stable. So, presumably, electrons moving through a crystal preserve their spins over many lattice sites. Besides, the Ising type coupling between itinerant electrons was already studied in the framework of the $s-d$ model $^{17}$.

Here we show that the model (1) captures driving mechanismes of formation of stripe phases and other charge and/or magnetically ordered superstructures. The class of materials fitting this picture is quite big and encompasses conductors that are magnetically ordered in low temperatures, but with no sign of the Kondo effect (as the latter implies the spin-flip process). There is a huge number of such systems, including many compounds of lanthanides and actinides, where various complicated magnetic structures were detected ${ }^{18}$. However, the model we propose is primarily aimed to desrcibe systems that display both a charge and magnetic order. In particular, we expect that dopped systems with a large crystal field effect would be the best candidates. The dopped systems, as they are usually composed of ions with different occupancies of localized electrons (a mixed-valency regime) and because they usually contain band electrons. This category includes materials, where stripe phases were recently detected as e.g. $\mathrm{La}_{1.6} \mathrm{Nd}_{0.4} \mathrm{Sr}_{x} \mathrm{CuO}_{4}{ }^{19}, \mathrm{YBa}_{2} \mathrm{Cu}_{3} \mathrm{O}_{6+x}{ }^{12}, \mathrm{Bi}_{2} \mathrm{Sr}_{2} \mathrm{CaCu}_{2} \mathrm{O}_{8+\delta}{ }^{14}$ or $\mathrm{La}_{1.5} \mathrm{Sr}_{0.5} \mathrm{NiO}_{4}{ }^{20}$. The behaviour of systems like those was already analyzed theoretically 
on a basis of the extended Hubbard model ${ }^{21,22}$ and the $t-J$ model $^{23}$.

On the other hand, a strong crystal field makes a flip of magnetic moment of an ion difficult. Consequently, a process of a simultaneous flip of spins of a localized and an itinerant electron becomes rare. This is another justification of taking into account only the Ising-type, instead of isotropic Heisenberg-type coupling in the model.

A major interaction omitted in the Hamiltonian (1) is the Hubbard-type interaction between spin-up and spin-down electrons. An inclusion of that term would make it intractable rigorously for arbitrary values of the coupling constants, but once again, one can imagine a simple justification for the omission. Namely, it is assumed here that the strength of the on-site interactions between two particles abides by the following hierarchy: a) the largest one - when the both particles are localized, b) an intermediate one - if one of the particles is localized and the other is itinerant, c) a negligible one - when the both particles are itinerant. This hierarchy may be summarized by a rather natural rule: the longer time particles occupy the same site, the more important becomes interaction between them. We point out here that the cases a) and b) are treated exactly within the model we propose. Obviously, there are no obstacles to include various interactions neglected in the Hamiltonian (1) as perturbations. On the other hand, the simplicity of the model (1) makes it attractive for studies using various techniques, both analytical and numerical.

An influence of the Hubbard interaction $U_{d d}$ between spin-up and spin-down itinerant electrons on an arrangement of the localized electrons is not known in a general case, but for $U_{d d}$ small we don't expect any dramatic changes of the phase diagram. However, if $U_{d d}$ is large, the situation may be different. For example, for half-filling (one itinerant electron per site) the itinerant electrons are ordered aniferromagnetically in the large $U_{d d}$ limit and they impose the same simple type of order to the localized electrons. Then, if there is one localized electron per site, the configuration of the localized electrons will be antiferromagnetic, i.e. the same as in the case studied in the current paper. If, however, the density of localized electrons is equal to $1 / 2$, the ground-state configuration D3 (see Fig. 2c) of the model (1) will have higher energy than the phase corresponding to the configuration $b$ displayed in Fig. 2a. So, if the Hubbard interaction is taken into account transformations of groundstate configurations of localized electrons are expected for some pairs of densities of electrons $\left(\rho_{d}, \rho_{f}\right)$ but not for the whole range of their values.

In the current paper we study the ground-state phase diagrams of the model (1) on the square lattice. However, the method we use can be applied to various types of onetwo- or three - dimensional lattices. The studies are based on the method of restricted phase diagrams ${ }^{3}$ constructed in the grand canonical ensemble (in the plane $\left(\mu_{d}, \mu_{f}\right)$ - see Fig. 1a) and then translated into a canonical ensemble diagram (in the plane $\left(\rho_{d}, \rho_{f}\right)$ - see Fig. 1b). Working within the framework of a grand canonical ensemble first assures the thermodynamic stability conditions are fulfilled (see Ref. ${ }^{24}$ ).

Another important reason for using the chemical potentials $\mu_{d}$ and $\mu_{f}$ in the model (1) is a possibility of changing and adjusting the electron's occupation numbers. Then, although the model does not contain a hybridization term, suitable changes of the chemical potentials or the position of the $E_{f}$ level can produce appropriate changes of the occupation numbers. In particular, fixing a total number of electrons enables us to study classical intermediate valence states, where some localized electrons change their occupancy to move into the delocalized states, conserving the total number of electrons. Of course, a change of 
the chemical potentials or $E_{f}$ cannot evoke a change of the electron states but only their occupation numbers.

Previous work on the spinless FKM has revealed a rich structure of the phase diagrams ${ }^{3,4}$. A number of charge ordered superstructures has been found, including stripe and nonstripe phases. However, so far little is known about magnetic structures accompanying those of charge ones, except for the limit of large $J$, where the ferromagnetic order was proven to exist for $\rho_{d}<\rho_{f} \leq 1^{15}$. As far as we know, the present study represents the first analysis of both charge and magnetic ordering in the framework of a generalized Falicov-Kimball based model for finite $U$ and $J$ values.

In the next section we explain how the calculations were carried out. The restricted phase diagrams are presented and described in the section III. The last section contains summary and conclusions.

\section{METHOD OF CALCULATION}

Here we consider all possible configurations of the localized $f$-electrons (including their spins), for which the number of sites per unit cell $N_{0}$ is less or equal to $N_{c}=4$. Taking into account all allowable sizes and shapes of the unit cells, as well as all relevant translational vectors, we found 47 distinct configurations. Some of them, representing phases with the smallest periods (up to 2 sites in an unit cell) and those with higher periods that are analyzed in this paper, are displayed in Fig. 2. The presentation of the configurations is chosen in such a way that make an easy observation of their transformations, starting from the ferromagnetic phase $(\mathrm{F})$ and ending either at the antiferromagnetic one (AF) (Confs. 1-4), or at the "empty" one (E) (Confs. D1-D5), in accordance with the diagrams given in Fig. 1.

For each periodic configuration in our trial set we performed the Fourier transformation of the Hamiltonian (1) and determined the electronic band structure for the conduction electrons. In other words, we solved the eigenvalue problem and found the eigenvalues $E_{\nu \sigma k}$, with branch index $\nu=1,2, \ldots, N_{0}$, spin index $\sigma$ and the Bloch wavevector $k=\left(k_{x}, k_{y}\right)$ (for more details see Refs. $\left.{ }^{3,4}\right)$. This required us to diagonalize up to $4 \times 4$ matrices and resulted in analytical formulae for at most 4 different energy bands, separately for spin-up and spindown electrons. (It is the main reason why the maximum size of the unit cell is limited to $N_{c}=4$ only, as the analytical formulae for phases with larger unit cells are not known in the general case.) In the simplest cases, related to 6 configurations with unit cells containing 1 or 2 sites (Confs. E, F, AF, a, b-see Fig. 2a and Conf. 3 - see Fig. 2b), the eigenvalues are given in the Appendix. For the remaining configurations, with unit cells containing 3 or 4 sites, the analytical formulae are quite long and for that reason are not displayed here.

Having exact formulae for the energy spectra $E_{\nu \sigma k}$ all quantities of interest can be calculated from the densities of states of spin-up and spin-down electrons

$$
Z_{\sigma}(E)=\sum_{\nu} \int_{B Z} d k \delta\left(E-E_{\nu \sigma k}\right) .
$$

In particular, the electron densities

$$
\rho_{d \sigma}\left(\mu_{d}\right)=\int_{-\infty}^{\mu_{d}} Z_{\sigma}(E) d E
$$


and the total electronic energy per site

$$
E_{t o t}\left(\mu_{d}\right)=\int_{-\infty}^{\mu_{d}} E\left(Z_{\uparrow}(E)+Z_{\downarrow}(E)\right) d E
$$

as a function of the chemical potential (the Fermi energy) $\mu_{d}$. The energy per site in the ground canonical ensemble (the Gibbs thermodynamical potential) is defined as

$$
E_{g c}=E_{t o t}-\mu_{d}\left(\rho_{d \uparrow}+\rho_{d \downarrow}\right)-\mu_{f}\left(\rho_{f \uparrow}+\rho_{f \downarrow}\right) .
$$

For the simplest configurations E, F and AF the quantities can be obtained analytically, but the expressions are rather complicated (they are given in terms of special functions). This is way in our calculations we employ a Brillouin zone grid of $100 \times 100$ momentum points for each bandstructure. At each discrete momentum point of the Brillouin zone we get at most 4 eigenvalues. Then the eigenvalues of the bandstructure are summed to determine the ground-state energy for each number of conduction electrons. The Gibbs thermodynamical potential is calculated for all possible values of the chemical potentials of the conduction and localized electrons through the formula

$$
E_{g c}\left(\left\{w_{i}\right\}\right)=\sum_{\sigma}\left(\frac{1}{10000 N_{0}} \sum_{\epsilon_{j \sigma}<\mu_{d}} \epsilon_{j \sigma}\left(\left\{w_{i}\right\}\right)-\mu_{d} \rho_{d \sigma}-\mu_{f} \rho_{f \sigma}\right),
$$

where $w_{i}=0$ or $\uparrow$ or $\downarrow$ characterizes the localized electron state at site $i$, and the symbol $\epsilon_{j \sigma}\left(\left\{w_{i}\right\}\right)$ denotes the energy eigenvalues of the bandstructure for the given configuration of localized electrons $\left\{w_{i}\right\}$. The ground canonical phase diagram is constructed in the plane of $\left(\mu_{d}, \mu_{f}\right)$. We directly compare the ground state energies of all phases from the trial set, and select the lowest one. Finally, the grand-canonical diagram is translated to a canonical phase diagram for arbitrary densities $\rho_{f}=\rho_{f \uparrow}+\rho_{f \downarrow}$ and $\rho_{d}=\rho_{d \uparrow}+\rho_{d \downarrow}$ of the $f-$ and $d$-electrons, respectively. This procedure assures thermodynamical stability of all phases (both periodic and their mixtures) present in the resulting canonical phase diagrams ${ }^{24}$.

\section{RESULTS AND DISCUSSION}

The grand canonical phase diagrams plot phase boundaries, which divide the plane of chemical potentials into domains, in each of which a single localized electron configuration forms the ground state. The general structure of the diagrams (Fig. 1a) is similar to that found for the spinless $\mathrm{FKM}^{3}$. It is relatively simple for large $U$ and more complex for small $U$. However, since the spin degeneracy is now lifted, new domains related to various magnetic arrangements having the same distribution of localized particles appear. In particular, the upper region corresponding to the full configuration in the spinless case is now split into a set of phases, ranging from $\mathrm{F}$, through ferri- and various complex antiferromagnetic to the simplest AF phase; see Figs. 1, 2a,b. The lower region corresponds to the empty configuration ( $\mathrm{E}$ - see Fig. 2a), and the largest of the remaining regions is the diluted antiferromagnetic phase (D3 - see Fig. 2c).

In the case of $U=8, J=0.5$ the central portion of the diagram is divided into diagonal stripes in which various periodic configurations of localized electrons are ground states. The sequence of the localized electron densities, reading from the left to right at fixed $\mu_{f}$ is $3 / 4$, 
2/3, 1/2, $1 / 3$ and $1 / 4$ (Confs. D1-D5, see Fig. 2c), the $\rho_{f}=1 / 2$ phase (D3) being the largest region.

In the corresponding canonical diagram (in the plane $\left.\left(\rho_{d}, \rho_{f}\right)\right)$ given in Fig. 1b all these phases are situated along the line $\rho_{f}+\rho_{d} / 2=1$. Movement along that line (starting from F) corresponds to a dilution of the ferromagnetic state by a gradual replacement of the localized electrons by itinerant ones in such a way, that each localized electron is replaced by two itinerant ones. This is different from the spinless case, where periodic phases are located along the line $\rho_{f}+\rho_{d}=1$. The difference is related to the fact, that now both spin up and spin down itinerant electrons can independently occupy each site, so the total density $\rho_{d}$ can take any value between 0 and 2 .

The analysis of the canonical phase diagram displayed in Fig.1b shows a strong tendency to antiferromagnetic order in the region of densities $\rho_{d}$ around 1 (half-filling) and to the ferromagnetic order outside that region. Indeed, both the phases D2 and D3 (see Fig. 2c) situated along the line $\rho_{f}+\rho_{d} / 2=1$ and those represented by the configurations no. 3,4 and AF (see Figs. 2a,b) situated along the line $\rho_{f}=1$ are ordered antiferromagnetically.

For intermediate values of the density $\rho_{d}$ and $\rho_{f}=1$ both antiferro- and ferrimagnetically ordered phases are found (Conf. 1 and 2 - see Fig. 2b). These phases represent consecutive stages of a reconstruction process when $\mathrm{F}$ transforms into the simplest $\mathrm{AF}$ with an increase of $\rho_{d}$.

On the other hand, only ferro- (F, D1, D4, D5 - see Figs. 2a,c) and antiferro- (D2, D3 - see Fig. 2c), but not ferrimagnetically ordered phases were found along the line $\rho_{f}+\rho_{d} / 2=1$. Moving along that line (in Fig. 1b) from the left-upper corner $\left(\rho_{d}=0, \rho_{f}=1\right)$ to right-lower one $\left(\rho_{d}=2, \rho_{f}=0\right)$ one can notice that the process of dissolution of the full ferromagnetic phase overlaps with the tendency to antiferromagnetism inside a region around half-filling.

The picture becomes more complicated as $U$ is reduced. An example is the case of $U=2$ and $J=0.5$ (not displayed here), where complex structures of the diagrams are found both in the plane of chemical potentials $\left(\mu_{d}, \mu_{f}\right)$ and densities $\left(\rho_{d}, \rho_{f}\right)$. First of all, more phases appear in the diagrams (20, versus 12 in the case of $U=8$ ) and the domains occupied by the phases in the central region are no longer diagonal stripes parallel to each other, but have a less regular structure.

The phases from the central region are distributed in different sectors of the plane $\left(\rho_{d}, \rho_{f}\right)$ in the corresponding canonical phase diagram. Only three periodic phases are found along the line $\rho_{f}+\rho_{d} / 2=1:$ D3, D5 and D1a - see Figs. 2c,d. Two of them (D3 and D5) already appeared in the $U=8$ case, but the ferrimagnetically polarized D1a phase replaces the ferromagnetic D1 (compare Figs. 2c,d). This fact illustrates a possibility of phase transitions from one magnetic structure to another with a change of $U$.

Another characteristic feature of the periodic phases found in the $U=2$ diagram is their stability with respect to finite intervals of the density $\rho_{d}$. This means that the Fermi level of the corresponding phase lies inside its energy band, so those phases are conducting. This is just the opposite to the situation found in the $U=8$ diagram (Fig.1b), where the phases D1-D5 are ground states for fixed values of itinerant electron densities $\rho_{d}$, corresponding to insulating states (the Fermi levels are situated inside their energy gaps).

Presumably, if we increase the maximum size of unit cells $N_{c}$, then phases with larger periods will enter the phase diagram in such a way, that intrevals of the density $\rho_{d}$ where 
low-period phases are stable will decrease. It is not clear from our studies if the intervals shrink to single values or to finite intervals of the density in the full phase diagram. Perhaps the first scenario occurs for some phases and the other for the rest.

A common feature of the canonical diagrams for $U=8$ and $U=2$ is that in both cases the fully occupied phases with $\rho_{f}=1$ (Confs. F, 1-4, AF) are the same and have identical positions. It appears that a distribution of these phases is symmetric with respect to half-filling $\rho_{f}=1$ and it does not depend on $U$, but merely on $J$.

Phases with $\rho_{f}=1$ that appear in the diagrams shown in Fig. 1 seem to be stable within finite intervals of $\rho_{d}$. This means that their ground states can be conducting. In particular, antiferromagnetic conductors appear to be possible ground states for certain electron concentrations off of half-filling. However, this is probably an artifact due to the restriction imposed by the maximum period of configuration in the trial set. If one takes into account configurations with larger periods then, presumably, some of them will enter the phase diagrams in such a way, that more and more phases will be stable merely within energy intervals lying inside one of their energy gaps. Therefore, one expects that the full phase diagram will contain many periodic phases in the insulating states, and only a part of them, especially those having gapless energy spectra, as e.g. those represented by the Conf. $\mathrm{E}$ and $\mathrm{F}$, will be conducting.

A similar situation was observed for the spinless FKM, where large period phases occupy regions of the phase diagram located outside of the energy gaps of phases having low periods $^{2,3}$.

\section{SUMMARY AND CONCLUSIONS}

A simple model capable of describing both charge and magnetic structures of localized electrons, as well as itinerant band magnetism, was introduced and investigated on a square lattice. Restricted ground state phase diagrams were constructed for intermediate values of the coupling parameters. Various types of charge and magnetically ordered phases were detected for a range of band fillings, illustrating e.g. consecutive stages of transformation of $\mathrm{F}$ to $\mathrm{AF}$ with an increase of the band filling.

It is remarkable, that the results presented here are consistent with those found for the Hubbard model in the large $U$ limit $^{25}$ and also with those obtained in Ref. ${ }^{15}$, where - in turn - the limit of large $J$ was investigated, as in all these cases one gets the simplest AF at half-filling and F far away from that limit. However, according to Ref. ${ }^{15}$ and Ref. ${ }^{25}$ the ferromagnetic ground state extends to all band fillings but half-filling, which is consistent with the Nagaoka theorem ${ }^{26}$. This, of course, is a consequence of investigation of the large $U$ or $J$ limit.

Here, instead, we provide results that give an opportunity to determine the upper limit for the density of itinerant electrons below which $\mathrm{F}$ can be stable for finite $U$ and $J$. Indeed, a direct comparison of energies of phases from the restricted set (they were calculated rigorously by using the exact analytical formulae for their energy spectra) shows that $\mathrm{F}$ can be stable for $\rho_{d} \leq 0.131$ ( or $\rho_{d} \geq 2-0.131$ ) if $J=0.5$ and for $\rho_{d} \leq 0.233$ (or $\rho_{d} \geq 2-0.233$ ) if $J=1$. These results complete those given in Ref. ${ }^{15}$, where no critical value for $\rho_{d}$ below which $\mathrm{F}$ is stable was reported. From the theorems provided in Ref. ${ }^{15}$ it may be merely concluded, that $\mathrm{F}$ is stable for any $0<\rho_{d}<\rho_{f}$ if $\rho_{f}=1$ and $J$ is large enough. 
Since no restrictions were here imposed on values of $U$ or $J$, it was not a great surprise that other ordered phases than just the simplest AF and $\mathrm{F}$ have been detected for intermediate electron concentrations. In particular, various magnetically ordered phases were found for $\rho_{f}=1$ (when charge is distributed uniformly) and intermediate $U$ and $J$, illustrating a transformation of $\mathrm{F}$ to $\mathrm{AF}$ with an increase of band filling.

But what are the physical reasons and what are the driving forces that lead to the complex charge and/or magnetic arrangements? If one attempts to describe such behavior in terms of two-body forces, then interactions between more distantt than the nearest neighboring lattice sites needs to be exploited. The double exchange ${ }^{27}$ and RKKY interactions, derived from the correlated electron models by using perturbative methods, are prominent examples of such approaches.

On a basis of the present studies we conclude that the phenomenon of charge and/or magnetic ordering can be explained by a mutual adjustment of the distributions of the charges and spins of the itinerant and localized electrons. This idea is not new, since it constitutes a background for the famous density functional theory. The difference is that we already start from an effective Hamiltonian (represented here by (1)) and consider many lattice sites instead of focusing on the details of a charge distribution around a single site, as is commonly practiced in the ab initio calculations. This allows us to take into account the kinetic energy of the itinerant electrons and to extract the most essential information about the system under investigation.

Indeed, one needs to investigate large enough areas accessible for itinerant electrons, as their total energy depends on the distribution of the localized ones over many lattice sites. If the latter forms a charge and/or magnetically ordered structure, then the mean values of the charge density distribution and/or the spin polarization of the moving electrons also adopts a suitable structure. It is clear that in the simplest case of a ferromagnetic metal, the densities of itinerant spin-up and -down electrons spread out uniformly over the whole lattice, but their values on each site are different, i.e. the moving electrons are polarized. But in the general case the distributions of itinerant spin-up and -down electrons are inhomogeneous and they may differ one from another.

Such an inhomogeneous distribution means that the itinerant electrons have some more preferable routes of travelling though the crystal (the routes may be different for the spin-up and -down electrons). So one can notice here something like a traffic self-regulation, where the system of localized electrons orders in such a way that the itinerant electrons would have as much freedom to move as possible. This of course is governed by the quantum mechanical laws through a minimization procedure of the total energy, which depends not only on the coupling constants, but also on the densities of the electrons.

It is interesting that many of the stable structures are axial (the lines of equivalent sites are parallel to the lattice axis) or diagonal (the corresponding lines are oriented along the $(1,1)$ direction) stripes. Axial stripes are predominant for rather small band fillings, i.e. for small densities of itinerant electrons, and the diagonal stripes are found close to the half-filling. The same picture was already observed for the spinless FKM ${ }^{4}$.

This stripe-type ordering means that the itinerant electrons prefer to move along simple, one-dimensional channels. Has this observation anything to do with, or can it shed light on a mechanism of the high $-T_{c}$ superconductivity? It is too early to answer this question, but the fact is that the stripe ordered phases have been detected in many high $-T_{c}$ materials. 
So further studies along the lines indicated here will be interesting.

Another point is that the detection of charge and magnetic phases other than $\mathrm{F}$ or AF provides an opportunity to describe various structures observed in many systems. Indeed, for many years various complex magnetic structures were observed in materials containing transition metals, rare-earths or actinides. Recently, thanks to new precise experimental techniques, stable charge superstructures have been also found in a number of compounds (see e.g. ${ }^{12}$ and the references given there). The model proposed here is capable to describe such structures in a simplified way but, of course, this work is merely the first step towards a complete analysis of the apparently very complicated processes occurring in real materials.

It is worthwhile to notice, that even though only the restricted phase diagrams were analyzed here, the conclusions are expected to also hold for the complete phase diagrams. This conjecture comes from the fact that the reported results are consistent with those obtained exactly in limiting cases and from a comparison with the data found previously for the spinless FKM, where an increase in the size of the allowed unit cells does not produce significant qualitative change in the phase diagram.

In closing we admit that it is clear that the simple effective model presented in this paper cannot describe many interesting phenomena observed in solids, like the Kondo effect or the superconductivity. However, since it is already a non-trivial model that can describe some of the phenomena, it can serve as a reference system for studies of more elaborate models of correlated electron systems. 


\section{ACKNOWLEDGMENTS}

I would like to thank Prof. G. Czycholl, Prof. P. Fulde, Dr. E. Runge, Prof. J. Spałek, Dr. J. Ulner, Dr. P. Wróbel and Dr. A. N. Yaresko for useful discussions on the topics related to this paper and prof. J. K. Freericks for helpful remarks and critical reading of the manuscript. This work was partially supported by The Max Planck Institute for Complex Systems in Dresden, Germany.

\section{APPENDIX A}

Below are given exact formulae for the energy spectra $E_{j k \uparrow}$ of the spin-up itinerant electrons in the simplest cases of period 1 and 2 configurations for the localized electrons (Conf. E, F, AF, 3, $a, b$ - see Figs. 2a,b). The corresponding spectra of the spin-down electrons can be obtained by taking the opposite signs of parameter $J$.

$$
\begin{aligned}
E_{1 k \uparrow}^{E}\left(k_{x}, k_{y}\right) & =2\left(\cos k_{x}+\cos k_{y}\right) \\
E_{1 k \uparrow}^{F}\left(U, J, k_{x}, k_{y}\right) & =U-J+2\left(\cos k_{x}+\cos k_{y}\right) \\
E_{2}^{A F}\left(U, J, k_{x}, k_{y}\right) & =U \mp \sqrt{J^{2}+4\left(\cos k_{x}+\cos k_{y}\right)^{2}} \\
E_{2}^{3} k \uparrow & \left(U, J, k_{x}, k_{y}\right)=U+2 \cos k_{y} \mp \sqrt{J^{2}+4\left(\cos k_{x}\right)^{2}} \\
E_{2_{2} k \uparrow}^{a}\left(U, J, k_{x}, k_{y}\right) & =\frac{1}{2}\left[U-J+4 \cos k_{y} \mp \sqrt{(U-J)^{2}+16\left(\cos k_{x}\right)^{2}}\right] \\
E_{\frac{1}{2} k \uparrow}^{b}\left(U, J, k_{x}, k_{y}\right) & =\frac{1}{2}\left[U-J \mp \sqrt{(U-J)^{2}+16\left(\cos k_{x}+\cos k_{y}\right)^{2}}\right]
\end{aligned}
$$




\section{REFERENCES}

${ }^{1}$ L. M. Falicov and J. C. Kimball, Phys. Rev. Lett. 22, 997 (1969).

${ }^{2}$ J. Lach, R. Łyżwa and J. Jȩdrzejewski, Acta Phys. Pol. A 84, 327 (1993); Phys. Rev. B 48, 10783 (1993).

${ }^{3}$ G. I. Watson and R. Lemański J. Phys. Condens: Matter 7, 9521 (1995).

${ }^{4}$ R. Lemański, J. K. Freericks and G. Banach, Phys. Rev. Lett. 89, 196403 (2002); J. Stat. Phys. 116, 699 (2004).

${ }^{5}$ U. Brandt, A. Fledderjohann and G. Hülsenbeck, Z. Phys. B 81, 409 (1990); U. Brandt and A. Fledderjohann, Z. Phys. B 87, 111 (1992).

${ }^{6}$ P. Farkašovsky̌, Phys. Rev. B 54, 11261 (1996).

7 J. K. Freericks and V. Zlatić, Phys. Rev. B 58, 322 (1998).

${ }^{8}$ V. Zlatić , J. K. Freericks, R. Lemański and G. Czycholl, Phil. Mag. B 81,1443 (2001);

${ }^{9}$ J. K. Freericks and V. Zlatić, Rev. Mod. Phys. 75, 1333 (2003).

10 J. Jȩdrzejewski and V. Derzhko, Physica A 317, 227 (2003).

${ }^{11}$ R. Lemański and J. Wojtkiewicz, phys. stat. sol. (b) 236, 408 (2003).

${ }^{12}$ H. A. Mook, P. Dai and F. Doğan, Phys. Rev. Lett. 88, 097004 (2002).

${ }^{13}$ Y. Ando, K. Segawa, S. Komiya and A. N. Lavrov, Phys. Rev. Lett. 88, 137005 (2002).

${ }^{14}$ C. Howald, H. Eisaki, N. Kaneko, M. Greven and A. Kapitulnik, Phys. Rev. B 67, 014533 (2003).

15 J. Fröhlich and D. Ueltschi, cond-mat/0404483.

16 S. V. Vonsovsky, Magnetism, New York, Wiley, 1974.

${ }^{17}$ B. M. Letfulov and J. K. Freericks, Phys. Rev. B 64, 174409 (2001).

${ }^{18}$ P. Santini, R. Lemański and P. Erdös, Adv. in Phys. 48, 537 (1999).

19 J. M. Tranquada, B. J. Sternlieb, J. D. Axe, Y. Nakamura and S. Uchida, Nature 375, 561 (1995).

${ }^{20}$ R. Kajimoto, K. Ishizaka, H. Yoshizawa and Y. Tokura, Phys. Rev. B 67, 014511 (2003).

${ }^{21}$ J. Zaanen and A. M. Oleś, Ann. Physik 5, 224 (1996).

${ }^{22}$ D. Góra, K. Rościszewski and A. M. Oleś, Phys. Rev B 60, 7429 (1999).

${ }^{23}$ C. S. Hellberg and E. Manousakis, Phys. Rev. Lett. 83, 132 (1999).

${ }^{24}$ Z. Gajek, J. Jȩdrzejewski and R. Lemański, Physica 223 A, 175 (1996).

${ }^{25}$ P. W. Anderson, Phys. Rev. 14, 115 (1959).

${ }^{26}$ Y. Nagaoka, Phys. Rev. 147, 392 (1966).

${ }^{27}$ C. Zener, Phys. Rev. 82, 403 (1951); P. W. Anderson and H. Hasegawa, ibid 100, 675 (1955). 


\section{FIGURES}

FIG. 1. The restricted ground-state phase diagrams for $U=8$ and $J=0.5$ : (a) the ground canonical phase diagram, i.e. in the plane of chemical potentials $\left(\mu_{d}, \mu_{f}\right)$, (b) the canonical phase diagram, i.e. in the plane of densities $\left(\rho_{d}, \rho_{f}\right)$. F, AF and E refer to the ferromagnetic, antiferromagnetic and empty (i.e. without localized f-electrons) phases, respectively. Periodic phases with $\rho_{f}=1$ are denoted by the numbers $1-4$ (see Fig. 2b), and those with $\rho_{f}+\rho_{d} / 2=1$ by the symbols D1-D5 (see Fig. 2c). (The domains D2 and D4 are very thin, therefore their boundaries are so close to each other that they have the appearence of a single thick line.) In the case (a), the phases occupy finite size domains and mixtures refer to the lines that separate the domains. In the case (b), the phases are ground states only on the bold straight-line segments or at single points. Outside these straight line segments or points there are mixtures of periodic phases that have lower energy than any periodic phase taken from the restricted set. The small vertical straight line segments crossing the $\rho_{f}=1$ line mark limits of $\mathrm{F}$. The diagonal line $\rho_{f}+\rho_{d} / 2=1$ is only a visual guide.

FIG. 2. Pictures of configurations of the localized electrons representing phases discussed in the paper. The $\uparrow(\downarrow)$ refer to sites occupied by a localized electron with spin up (down) respectively, and the dots denote sites not occupied by the localized electrons. The shaded region in the lower left corner shows the unit cell, and line segments show the translation vectors that are used to tile the two-dimensional plane. Panel (a) shows the simplest configurations of the localized electrons containing 1 or 2 sites per unit cell. Three of them (E - empty, F - ferromagnetic and AF antiferromagnetic) appear in the phase diagrams and the last two ( $a$ and $b)$ are not present in the diagrams. Panel (b), the configurations 1-4 with $\rho_{f}=1$, which are found on the restricted phase diagrams for $U=8$ and $J=0.5$ between $\mathrm{F}$ and AF. Panel (c), the configurations D1-D5 with $\rho_{f}<1$, which are found in the restricted phase diagrams for $U=8$ and $J=0.5$ between the $\mathrm{F}$ and E phases; on the canonical phase diagram (Fig. 1b) they appear along the diagonal $\rho_{f}+\rho_{d} / 2=1$. Panel (d), the configurations with $\rho_{f}=1$ (Confs. 1a, 5), that are found between the $\mathrm{F}$ and AF phases in the restricted phase diagrams for $U=8$ and $J=1.0$, but are not found on the diagrams for $U=8$ and $J=0.5$ : Conf. 1a replaces Conf. 1, whereas Conf. 5 appears between Conf. 4 and AF. The configuration D1a with $\rho_{f}=0.75$ is found on the restricted phase diagrams for $U=2$ and $J=0.5$ instead of D1 (see Fig. 2b). 

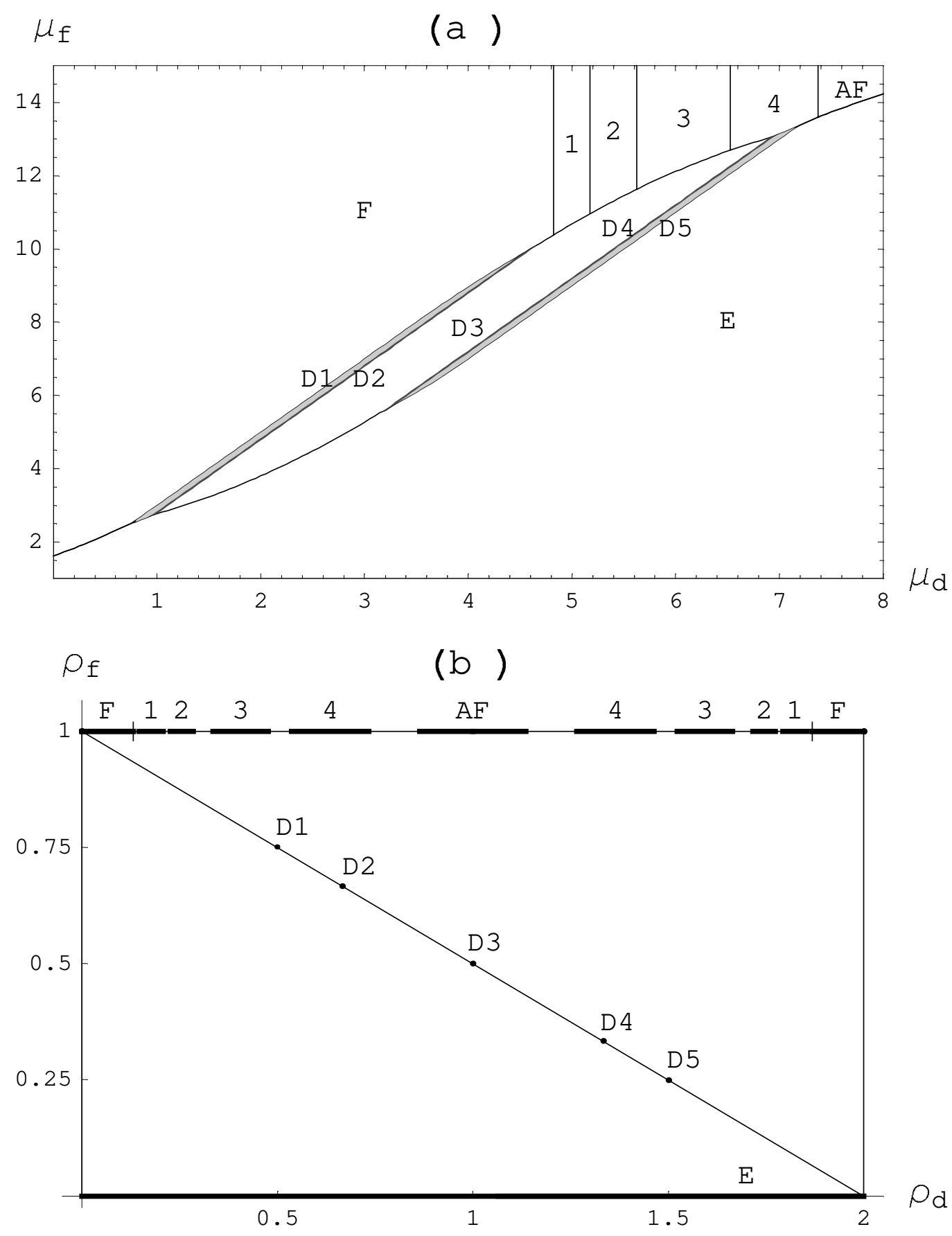
. . . . . . $1 \uparrow \uparrow \uparrow \uparrow \uparrow \uparrow \uparrow$

$\uparrow \quad \downarrow \quad \uparrow \quad \downarrow \quad \uparrow \quad \downarrow$

. . . . . $1 \uparrow \uparrow \uparrow \uparrow \uparrow \uparrow$

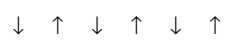

a

. . . . . . $1 \uparrow \uparrow \uparrow \uparrow \uparrow \uparrow$

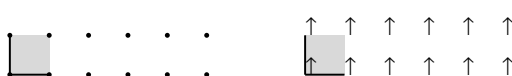

$\uparrow \downarrow \downarrow c c c c$

$\begin{array}{lllll}\downarrow & \uparrow & \downarrow & \downarrow & \uparrow \\ \llcorner & \downarrow & \downarrow & \uparrow & \downarrow\end{array}$

$\uparrow \cdot \uparrow \cdot \uparrow \cdot$

$\cdot \uparrow \cdot \uparrow \cdot{ }^{-} \cdot{ }^{-}$

$\uparrow \cdot \uparrow \cdot \uparrow \cdot$

$\uparrow \cdot{ }^{\uparrow} \cdot \dot{r}^{\uparrow} \cdot$

$\uparrow \cdot \uparrow \cdot \uparrow \cdot$

$\dot{\uparrow} \cdot \dot{\uparrow} \cdot \dot{\uparrow} \cdot$

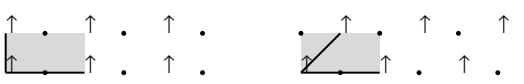




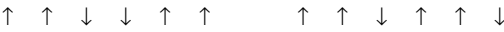

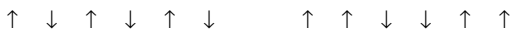

$\uparrow \quad \uparrow \quad \downarrow \quad \downarrow \quad \uparrow \quad \uparrow \quad c \quad \uparrow \quad \uparrow \quad \downarrow \quad \uparrow \quad \uparrow \quad \downarrow$

$\uparrow \quad \downarrow \quad \uparrow \quad \downarrow \quad \uparrow \quad \downarrow$

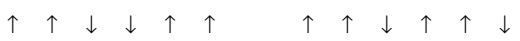

$\uparrow \downarrow \uparrow \quad \downarrow \quad \uparrow \quad \downarrow$

$\begin{array}{llllll}\uparrow & \uparrow & \downarrow & \downarrow & \uparrow & \uparrow \\ \uparrow & \uparrow & \downarrow & \downarrow & \uparrow & \uparrow\end{array}$

$\begin{array}{llllll}\uparrow & \uparrow & \downarrow & \uparrow & \uparrow & \downarrow \\ \uparrow & \uparrow & \downarrow & \uparrow & \uparrow & \downarrow\end{array}$

$\uparrow \quad \downarrow \quad \uparrow \quad \downarrow \quad \uparrow \quad \downarrow$

$\downarrow \quad \downarrow \quad \uparrow \quad \uparrow \quad \downarrow \quad \downarrow$

$\uparrow \quad \uparrow \quad \downarrow \quad \downarrow \quad \uparrow \quad \uparrow$

$\begin{array}{lllll}\downarrow & \downarrow & \uparrow & \downarrow & \downarrow \\ \qquad & \downarrow & \downarrow & \uparrow & \uparrow\end{array}$ 


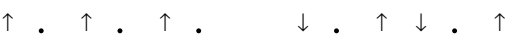

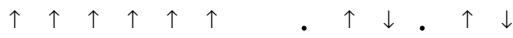

$\uparrow \cdot \uparrow \cdot \uparrow$

. $\uparrow \cdot \uparrow \cdot \uparrow$

$\uparrow \downarrow . \uparrow \downarrow$.

$\cdot \downarrow \cdot \downarrow \cdot \downarrow$

\begin{tabular}{llll}
$\uparrow$ & $\uparrow$ & $\uparrow$ & $\uparrow$ \\
$\uparrow$ & $\uparrow$ & $\cdot$ & $\uparrow$ \\
\hline & $\uparrow$ & $\uparrow$ & $\uparrow$
\end{tabular}

$\begin{array}{lllll}\downarrow & \cdot & \uparrow & \downarrow & \uparrow \\ \cdot \perp_{\downarrow} & \downarrow & \uparrow & \downarrow & \downarrow\end{array}$

$\begin{array}{llll}\uparrow & \uparrow & \bullet & \downarrow \\ \downarrow & \cdot & \downarrow & \downarrow\end{array}$

$\cdot \uparrow \uparrow \cdot \cdot$

$\uparrow \cdot \uparrow \cdot \uparrow \cdot$

.

$\left[\begin{array}{lllll}\uparrow & \uparrow & \bullet & \bullet \\ \downarrow & \downarrow & \cdot & \downarrow\end{array}\right.$

$\uparrow \cdot . \uparrow \cdot . \quad \cdot \uparrow \cdot \uparrow \cdot \uparrow$

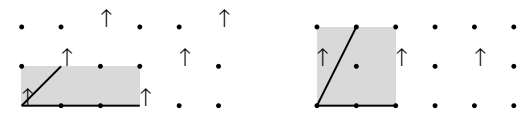


$\uparrow \quad \downarrow \quad \downarrow \quad \downarrow \quad \uparrow \quad \downarrow$

$\uparrow \downarrow \uparrow \downarrow \uparrow \downarrow$

$\uparrow \quad \downarrow \quad \downarrow \quad \downarrow \quad \uparrow \quad \downarrow$

$\downarrow \downarrow \downarrow \downarrow \downarrow \downarrow \downarrow$

$\uparrow \quad \downarrow \quad \downarrow \quad \downarrow d r$

$\downarrow \quad \uparrow \quad \downarrow \quad \uparrow \quad \downarrow \quad \uparrow$

$\uparrow \quad \downarrow \quad \downarrow \quad \downarrow \quad \uparrow \quad \downarrow$

$\begin{array}{llllll}\uparrow & \downarrow & \downarrow & \downarrow & \uparrow & \downarrow \\ \uparrow & \downarrow & \downarrow & \downarrow & \uparrow & \downarrow\end{array}$

$\begin{array}{llllll}\downarrow & \downarrow & \downarrow & \downarrow & \downarrow & \downarrow \\ \downarrow & \uparrow & \downarrow & \uparrow & \downarrow \\ \downarrow & \downarrow & \downarrow & \downarrow & \downarrow\end{array}$

$\uparrow \downarrow \uparrow, \uparrow \downarrow$

$\uparrow \quad \uparrow \downarrow \downarrow \downarrow$

$\uparrow \downarrow \uparrow, \uparrow \downarrow$

$\underset{\downarrow \uparrow}{\uparrow} \underbrace{\uparrow} \downarrow{ }^{\uparrow} \downarrow$ 